\title{
Efecto de dosis de nitrógeno sobre la producción de estaquillas de Eucalyptus grandis $\times$ E. urophylla en jardín clonal
}

\author{
Effect of nitrogen doses on the production of cuttings of \\ Eucalyptus grandis $\times E$. urophylla in clonal garden
}

Alex Machacuay ${ }^{1, \star}$ y Yury M. Llancari ${ }^{1}$

Recibido: 20 julio 2020 | Aceptado: 12 setiembre 2020 | Publicado en línea: 12 diciembre 2020 Citación: Machacuay, A; Llancari, YM. 2020. Efecto de dosis de nitrógeno sobre la producción de estaquillas de Eucalyptus grandis $\times$ E. urophylla en jardín clonal. Revista Forestal del Perú 35 (3, Número Especial): 5-17. DOI: http://dx.doi.org/10.21704/rfp.v35i3.1597

\begin{abstract}
Resumen
El objetivo del presente estudio fue evaluar el efecto de cinco dosis de nitrógeno $(\mathrm{N})$ en la producción y el intervalo de cosecha de estaquillas de cuatro clones de Eucalyptus x urograndis (Eucalyptus grandis $\times$ E. urophylla) a nivel de jardín clonal en la provincia de Satipo, departamento de Junín. Las dosis evaluadas fueron $0,28,32,36$ y 40 g.N $/ \mathrm{kg}$, las cuales se analizaron en parcelas conformadas por 30 plantas matrices cada una, siendo cada planta matriz una unidad experimental, los demás macronutrientes y micronutrientes se mantuvieron constantes. El promedio del número de estaquillas producidas para cada uno de los clones $(103,105$, H77 y 433) se determinó mediante cuatro colectas de estaquillas por cada planta matriz. Se empleó el análisis de variancia (ANVA) y la prueba comparativa de medias de Tukey a un nivel de significancia de 0,05. La productividad promedio de estaquillas a nivel de jardín clonal presentó influencia diferenciada por las dosis de $\mathrm{N}$ sobre los diferentes tipos de clones. Los clones 105, H77 y 433 mostraron un incremento significativo $(p \leq 0,05)$ de producción promedio de estaquillas, observándose los mejores valores promedios de productividad con las dosis de 36, 28 y 40 g.N $/ \mathrm{kg}$ respectivamente, sin embargo, para el clon 103 la anterior relación no es determinante para la elección de la dosis de $\mathrm{N}$ a aplicarse, al no presentar un incremento significativo en su producción media $(p>0,05)$. El intervalo promedio determinado para la cosecha de estaquillas de los clones fue de 14,25 días. Por último, el presente estudio mostró que los diferentes tipos de clones tienen diferentes requerimientos nutricionales para producción de estaquillas a nivel de jardín clonal.
\end{abstract}

Palabras clave: Eucalyptus x urograndis; semilla vegetativa; clones; jardín clonal

\footnotetext{
${ }^{1}$ Facultad de Ciencias Forestales, Universidad Nacional Agraria La Molina (UNALM), Av. La Molina s/n, La Molina, Lima, Perú.

* Autor de Correspondencia: amachacuayroman@gmail.com
} 
Efecto de dosis de nitrógeno sobre la producción de estaquillas de

\begin{abstract}
The aim of this study was to evaluate the effect of five nitrogen $(\mathrm{N})$ doses in the productivity and the interval of harvesting cuttings of four clones of Eucalyptus $\mathrm{x}$ urograndis (Eucalyptus grandis $\times$ E. urophylla) at a clonal garden level installed in Satipo, department of Junín. The doses evaluated were $0,28,32,36$ and 40 g.N/kg, which were analyzed in plots made up of 30 parent plants each, each parent plant being an experimental unit, the other macronutrients and micronutrients were kept constant. The average productivity of cuttings for each clone (103, 105, H77 and 433) was determined through four collections of cuttings for each parent plant. The analysis of variance (ANOVA) and Tukey's comparative test of means were used at a significance level of 0,05 . Clones $105, \mathrm{H} 77$ and 433 showed a significant increase $(p \leq 0.05)$ in average cuttings production, observing the best average productivity values with the doses of 36,28 and 40 g.N $/ \mathrm{kg}$ respectively, however, the previous relationship is not decisive for the selection of the $\mathrm{N}$ dose to be applied in clone 103, because it does not present a significant increase in its mean production $(p>0.05)$. The average interval determined for harvesting cuttings from the clones was 14.25 days. Finally, the present study showed that the different types of clones have different nutritional requirements for cuttings production at the clonal garden level.
\end{abstract}

Key words: Eucalyptus $\mathrm{x}$ urograndis; vegetative seed; clones; clonal garden; fertilization

\section{Introducción}

Según la FAO (2003), la madera es el único commodity a nivel mundial cuya demanda irá en aumento, al 2050 la población mundial alcanzará los 9300 millones de habitantes, con una demanda de 4500 millones de $\mathrm{m}^{3}$ de madera rolliza y cada persona consumirá $0,5 \mathrm{~m}^{3}$ de madera al año. Sin embargo, hasta ahora nuestras áreas forestales, a pesar de ocupar casi el $60 \%$ del territorio nacional, solo contribuyen con algo menos del 1\% del Producto Bruto Interno (SERFOR 2016), en comparación con países de la región que tienen menos extensión de bosques naturales, tales como Chile $(2,6 \%$ del PBI), Bolivia (2,7\%) y Ecuador (2,3\%) (Sánchez 2018).

Lo mencionado anteriormente evidencia la subutilización del alto potencial que tiene el país para el desarrollo de plantaciones forestales con fines comerciales, considerando, además, que las condiciones son favorables para el desarrollo de negocios forestales competitivos. Las últimas cifras oficiales indican que contamos con 1069768 hectáreas de plantaciones forestales a nivel nacional, de las cuales solo 50000 hectáreas son comerciales y abastecen apenas el 19\% de la demanda interna de madera (SERFOR 2019b), siendo estas su- perficies plantadas muy inferiores a la de países como Brasil (7,8 millones de hectáreas), Chile (2,4 millones de hectáreas) o Argentina (1,4 millones de hectáreas).

El potencial para plantaciones forestales de uso múltiple en la Amazonía peruana es de 6,3 millones de hectáreas, entre las cuales señalan tierras con algún título habilitante, las que están bajo dominio de comunidades nativas y campesinas, y las que no poseen derechos otorgados. Estas cifras ponen en evidencia la necesidad del desarrollo de plantaciones forestales que puedan atender la demanda nacional insatisfecha proyectada en $1455000 \mathrm{~m}^{3}$ de madera para el 2028, para lo cual se requeriría de 144116 ha de plantaciones forestales y 267 500 ha de bosques naturales SERFOR (2019a). Asimismo, la institución señala que en el año 2017 la demanda de productos de madera alcanzó $1304260 \mathrm{~m}^{3}$ siendo cubierta con la importación de $710083 \mathrm{~m}^{3}$, configurando una balanza comercial del sector forestal maderable que mantiene un déficit sostenido en promedio de 840 millones de USD/año.

Se debe considerar que, para lograr el éxito de una plantación forestal comercial se debe priorizar criterios de productividad, calidad y uniformidad que son significativamente ma- 
yores cuando los plantones proceden de semilla vegetativa. Asimismo, Quispe (2017) indica que la productividad de una plantación depende de tres factores: 1) material genético, 2) calidad de sitio, y 3 ) manejo silvicultural; siendo la incidencia del primero del 30 al $50 \%$. Por esta razón, resulta importante la producción de plantas de alta calidad genética, para lo cual se requiere contar con la infraestructura necesaria y el manejo correspondiente.

En ese sentido, es fundamental iniciar con la instalación de centros de producción de material genético de alta productividad en zonas estratégicas del país, siendo la selva central un área importante de producción maderera según el estudio de corredores económicos forestales realizado por el SERFOR (Quispe 2017). En los últimos 20 años, los jardines clonales tuvieron una evolución significativa en la reducción del área, aumento de la productividad $\left(\mathrm{m}^{3} / \mathrm{ha} /\right.$ año) y reducción del tamaño de la estaquilla (Higashi et al. 2002). Inicialmente, los jardines clonales eran plantados en una razón de 1:100, es decir, para plantar 100 ha era necesario 1 ha de jardín clonal (Campinhos 1983); pasando posteriormente a una razón de 1:525 (Higashi et al. 2000). Estos sistemas de jardines clonales aumentan la producción de plantas clonales de alta calidad genética como lo desarrollado en la región de Aracruz/ES - Brasil, empleando un método de plantío de mayor densidad para los jardines clonales, con 40000 plantas/ ha (Campinhos 1983). Asimismo, otros países como Chile, Argentina, Uruguay, España, Portugal, Marruecos y Sudáfrica también desarrollaron estos sistemas de jardines clonales.

En los jardines clonales se tiene la colección completa de árboles plus seleccionados a través de un proceso de mejoramiento genético, generalmente mediante la hibridación de dos especies compatibles y con características sobresalientes para un determinado fin comercial. Cada árbol plus es propagado a partir de brotes en el tocón u otras partes vegetativas del árbol, todas y cada una de las estaquillas que se logren reproducir de un mismo árbol plus son copias genéticamente idénticas y se les denomina rametos; y a estos se les identifica con el código del árbol plus (Badilla y Murillo 2005). Asimismo, los mismos autores mencionan que para tener una producción de estaquillas constante en el jardín clonal es importante tener un programa de fertilización para manejar la nutrición de las plantas, de esta manera poder asegurar una mayor cantidad y calidad de material vegetativo para la producción de plantas en viveros forestales mediante técnicas de estaquillado. El estado nutricional es un factor que influye directamente en la productividad de las plantas matrices, enraizamiento de las estaquillas y calidad de plántulas, ya que los macro y micronutrientes están involucrados en procesos bioquímicos y fisiológicos vitales de la planta (Paula 2000). Así también, Terbulino et al. (2015) señala respecto al nitrógeno $(\mathrm{N})$ que por ser parte de innumerables compuestos y estar directamente relacionado con la capacidad fotosintética de plantas, puede influir significativamente en la producción de estaquillas. Por lo cual resulta importante contar con estudios de productividad de estaquillas y programas de fertilización en diferentes condiciones de sitio para ofertar material vegetativo de alta productividad a proyectos forestales.

El híbrido Eucalyptus x urograndis (Eucalyptus grandis $\mathrm{x} E$. urophylla) es un recurso importante, debido a su aprovechamiento industrial en la obtención de celulosa y hemicelulosa para la fabricación de papel en varias partes del mundo (Martínez et al. 2005), y gracias a las características de su madera ha sido utilizada para postes de alumbrado, trozas para aserrados, puntales para construcción civil, pisos de parquet, construcción de muebles, soportes en minas, tableros de fibras, biomasa para energía, tutores para cultivos agrícolas, construcción de casas y cercos (Sánchez 2018).

El presente estudio tuvo como objetivo evaluar la producción de estaquillas a nivel de jardín clonal de cuatro clones de Eucalyptus $\mathrm{x}$ urograndis en la zona de Satipo, departamento de Junín, bajo el efecto de cinco dosis diferentes de nitrógeno y manteniendo los otros nutrientes constantes, con la finalidad de determinar qué clones presentan mayor productividad de estaquillas y en qué intervalo de cosecha. 
Efecto de dosis de nitrógeno sobre la producción de estaquillas de

Eucalyptus grandis $\times$ E. urophylla en jardín clonal

\section{Materiales y Métodos}

\section{Lugar de ejecución}

El estudio se realizó en un jardín clonal con cuatro clones de Eucalyptus x urograndis, en el distrito de Pangoa, provincia de Satipo, departamento de Junín. El área de ejecución está ubicada a una latitud de $11^{\circ} 29^{\prime} 36,7^{\prime \prime} \mathrm{S}$ y longitud $74^{\circ} 30^{\prime} 11,9^{\prime \prime} \mathrm{W}$ y a una altitud de 1032 m.s.n.m. La localidad presenta una temperatura promedio de $23,5^{\circ} \mathrm{C}$ y una precipitación promedio de $1618 \mathrm{~mm}$ al año.

\section{Materiales e insumos}

Se instalaron las parcelas de jardines clonales utilizando cuatro clones del híbrido Eucalyptus x urograndis, codificados como 103, 105, H77 y 433. Estos fueron establecidos sobre un suelo de clase textural arcilloso (Ar) y con un $\mathrm{pH}$ de 4,46. Los clones fueron adquiridos de la empresa Arborizaciones E.I.R.L., la cual cuenta con un vivero instalado en la localidad de Palca, Tarma, Junín. Asimismo, para los tratamientos de fertilización se utilizó nitrato de amonio $\mathrm{NH}_{4} \mathrm{NO}_{3}(33 \%$ de $\mathrm{N})$ como fuente de nitrógeno en dosis de 0, 28, 32, 36 y 40 g.N $/ \mathrm{kg}$, mientras que, para los macroelementos de fósforo y potasio se utilizó fosfato diamónico $(46 \%$ de $\left.\mathrm{P}_{2} \mathrm{O}_{5}\right)$ y cloruro de potasio $\left(60 \%\right.$ de $\left.\mathrm{K}_{2} \mathrm{O}\right)$ respectivamente, todos de la marca Inkafert fertilizantes $^{\mathrm{T} x}$, así como los micronutrientes $\mathrm{B}, \mathrm{Cu}$, $\mathrm{Fe}, \mathrm{Mn}, \mathrm{Mo}$ y Zn; los fertilizantes en mención se encuentran disponibles en la localidad y fueron empleados según las dosis recomendadas como adecuadas por Higashi et al. (2000) (Cuadro 1).

\section{Metodología}

\section{a. Criterios para la selección de plantas en vivero}

Para garantizar el éxito del jardín clonal se consideró los siguientes criterios de calidad en el proceso de selección de plantas:

- Índice de Esbeltez (IE); el cual relaciona la resistencia de las plantas con la capacidad fotosintética de la misma y se recomienda valores entre 5 y 10 como indicadores de una mejor calidad de planta (Toral 1997, citado por
Quiroz et al. 2009). El cálculo se realiza mediante la siguiente ecuación:

$$
I E=\text { Altura }(\mathrm{cm}) / \text { Diámetro basal }(\mathrm{mm})
$$

- Buen desarrollo radicular; las cuales deben ser abundantes, sobresalientes en la parte inferior del contenedor y no presentar enrollamiento. Además, de ser de color blanquecino revelando la verdadera calidad de la planta (Tello, J y Tello, E 2016).

- Buen estado fitosanitario; evitando seleccionar aquellas plantas que presenten manchas foliares, malformaciones, déficit nutricional y presencia de enfermedades, ya que, estas en campo definitivo representan riesgo y un gasto innecesario (Tello, J y Tello, E 2016).

Las plantas seleccionadas en tubetes de 53 cc fueron colocadas en contenedores de polipropileno de 187 cavidades y regadas previo a su traslado con abundante agua para contrarrestar el estrés hídrico. Asimismo, en la localidad donde se instaló el jardín clonal, fueron sometidas a un proceso de aclimatación bajo sombra, utilizando una malla Raschel al 50\% de sombra por un periodo de 20 días.

\section{b. Instalación de jardines clonales}

Para el establecimiento del jardín clonal se realizó la habilitación de terreno de forma manual, la limpieza del lugar se realizó a través de la utilización de mano de obra y uso de herramientas, como motosierra, hachas y machetes, eliminando todo tipo de malezas presentes. La preparación de suelo consistió en la confección de casillas de plantación en forma manual con pala plantadora en superficie de 40 $\times 40 \mathrm{~cm}$ y con una profundidad de $30 \mathrm{~cm}$ en lugares marcados previamente. Las plantas se instalaron a un distanciamiento de $1 \mathrm{~m} \times 1,5$ $\mathrm{m}$, luego de instalados se llevó un control de malezas de manera manual para eliminar todo aquel tipo de vegetación que pueda ser competitiva con la especie plantada. El proceso de instalación y manejo de jardín clonal se señala en el diagrama de la Figura 1.

\section{c. Aplicación de las dosis de fertilización}

Se realizó una fertilización de establecimiento a toda la población de estudio a la semana 


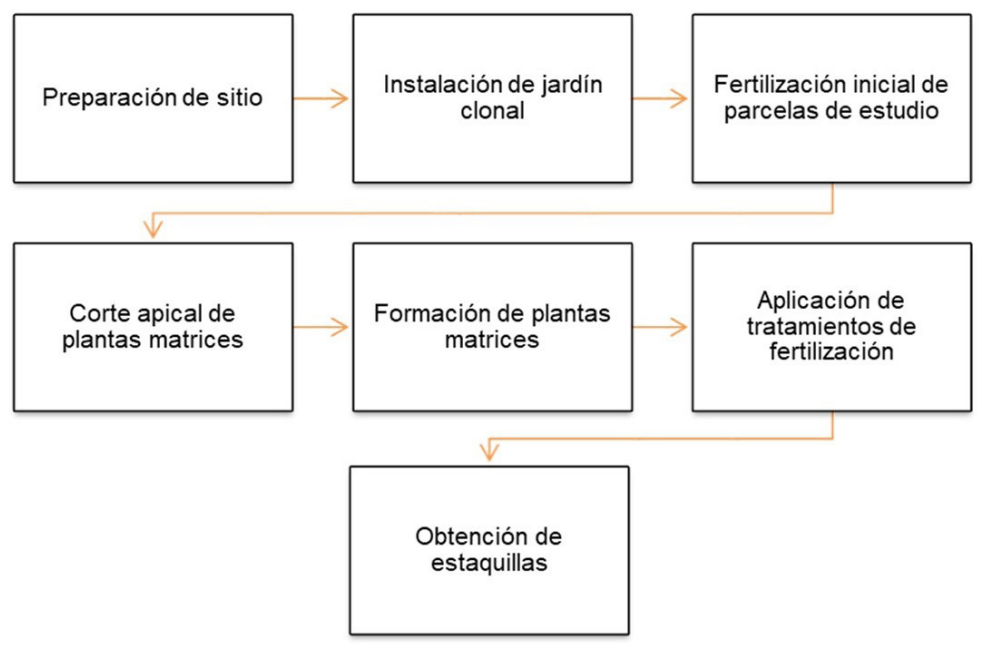

Figura 1. Diagrama del proceso de instalación y manejo de jardín clonal de Eucalyptus x urograndis.

de instalación del jardín clonal. Las dosis de fertilización estuvieron compuestas por macronutrientes y micronutrientes recomendados en la guía "Manejo en el primer año de una reforestación exitosa” elaborada por FONDEBOSQUE (s.f.). Las siguientes fertilizaciones fueron realizadas según las recomendaciones de Higashi et al. (2000) a excepción del N que fue el elemento a estudiar, la cual fue aplicada en dosis de $0,28,32,36$ y 40 g.N/kg. La primera aplicación de esta fertilización se realizó seguidamente del corte apical de las plantas, la cual se realizó una vez que las plantas superaron $1 \mathrm{~m}$ de altura. La segunda fertilización fue aplicada transcurrida la segunda colecta de estaquillas. Las fertilizaciones fueron aplicadas de forma manual a una distancia de $10 \mathrm{~cm}$ de la base de las plantas sobre una media luna.

\section{d. Criterios para la selección de estaquillas a cosechar}

La población de estudio estuvo conformada por 3332 plantas correspondientes a cuatro clones de Eucalyptus x urograndis (103, 105, H77 y 433), cada clon constituido por 833 plantas. Para cada tipo de clon se seleccionaron 5 parcelas de 30 plantas matrices cada una, donde se aplicaron los diferentes tratamientos con $\mathrm{N}$, las plantas restantes conformaron los bordes de las parcelas de evaluación. Para garantizar el éxito de las estaquillas en la etapa de enraizamiento en invernadero se consideró los criterios: tamaño de estaquilla entre 7 a 10 $\mathrm{cm}$ de longitud, que posean de 3 a 4 pares de hojas según lo recomendado por Terbulino et al. (2015), así como, tallos que presentan resistencia al contacto y buen estado sanitario de las estaquillas. Se realizó cuatro colectas de estaquillas en cada planta matriz de las parcelas de evaluación en intervalos determinados considerando los criterios mencionados anteriormente, esta repetitividad fue realizada con el fin de reducir el error de medición.

\section{e. Determinación del intervalo de cosecha de estaquillas}

Para la determinación de los intervalos de cosechas de estaquillas se contabilizaron los días transcurridos entre dos colectas consecutivas, estando este tiempo establecido por los criterios para la selección de estaquillas a cosechar.

f. Cálculo de la razón de rendimiento jardín clonal/plantación (ha)

Se consideró una densidad de 6666 plantas/ ha para el jardín clonal plantados con un distanciamiento de $1 \mathrm{~m} \times 1,5 \mathrm{~m}$ tomado como 
Efecto de dosis de nitrógeno sobre la producción de estaquillas de

\begin{tabular}{|c|c|}
\hline \multicolumn{2}{|c|}{ Dosis Adecuada } \\
\hline Nutrientes & Macronutrientes (g/kg) \\
\hline $\mathrm{N}$ & $28-40$ \\
\hline $\mathrm{P}$ & $2,5-4$ \\
\hline K & $15-30$ \\
\hline $\mathrm{Ca}$ & $5-7$ \\
\hline $\mathrm{Mg}$ & $2-3$ \\
\hline $\mathrm{S}$ & $2-2,5$ \\
\hline Nutrientes & Macronutrientes $(\mathrm{g} / \mathrm{kg})$ \\
\hline B & $35-70$ \\
\hline $\mathrm{Cu}$ & $8-15$ \\
\hline $\mathrm{Fe}$ & $101-220$ \\
\hline $\mathrm{Mn}$ & $250-500$ \\
\hline $\mathrm{Zn}$ & $30-60$ \\
\hline
\end{tabular}

Cuadro 1. Contenidos de macro y micronutrientes considerados adecuados para la producción de estaquillas a nivel de jardín clonal según Higashi et al. (2000).

referencia de Carvalho et al. (1991), citado por Higashi (2000). Además, se consideró una eficiencia de enraizamiento de $85 \%$ a nivel de invernadero referido por Quispe (2017) y una densidad de 1111 árboles/ha con un distanciamiento de $3 \mathrm{~m} \times 3 \mathrm{~m}$ para el cálculo de número de hectáreas posibles a plantar.

\section{g. Diseño experimental}

En el presente estudio se empleó el experimento bifactorial con diseño completamente aleatorizado (D.C.A.). Siendo el primer factor el tipo de clon y sus niveles $(103,105, \mathrm{H} 77$ y 433); el segundo factor la dosis de nitrógeno y sus niveles $(0,28,32,36$ y 40 g.N $/ \mathrm{kg})$. Se evaluó el efecto de estos factores en las plantas de Eucalyptus $\mathrm{x}$ urograndis a partir del número de estaquillas producidas por cosecha.

El análisis estadístico se realizó empleando el programa R 4.0.2, haciendo uso del modelo aditivo lineal:

$$
Y_{i j k}=\mu+\alpha_{i}+\beta_{j}+(\alpha \beta)_{i j}+\varepsilon_{i j k}
$$

Donde:

$\mathrm{Y}_{\mathrm{ijk}}=$ Cantidad de estaquillas obtenida con el clon $\mathrm{i}$, la dosis j y la repetición $\mathrm{k}$. $\mu=$ Es el efecto de la cantidad de estaquillas media general.

$\alpha_{i}=$ Es el efecto del clon $i$.

$\beta_{\mathrm{j}}=$ Es el efecto de la dosis $\mathrm{j}$.

$(\alpha \beta)_{\mathrm{ij}}=$ Es el efecto de la interacción entre el clon i y la dosis $\mathrm{j}$.

$\varepsilon_{\mathrm{ijk}}=$ Es el efecto del error experimental obtenido con el clon $\mathrm{i}$, la dosis $\mathrm{j}$ y la repetición $\mathrm{k}$.

El análisis de la variabilidad del efecto de los tratamientos sobre los resultados se realizó mediante el análisis de variancia (ANVA) y la prueba comparativa de medias de Tukey con un nivel de confianza con $95 \%$ de probabilidad $(p \leq 0,05)$.

\section{Resultados}

\section{Productividad de estaquillas}

La productividad media de estaquillas presentó diferencias significativas respeto al incremento de la dosis de N para los clones 105, H77 y 433. A excepción del clon 103, en el cual no se observó variación significativa en sus valores promedios de productividad.

Para el clon 105 los valores promedios de productividad obtenidos con las dosis de $36 \mathrm{y}$ 40 g.N $/ \mathrm{kg}$ son significativamente superiores a los promedios obtenidos con dosis inferiores. Sin embargo, no existen diferencias significativas entre los promedios de productividad obtenidos con las dosis de 36 y 40 g.N $/ \mathrm{kg}$.

Respecto al clon H77, muestra mayores valores promedios de producción a partir de la dosis 28 g.N/kg, no obstante, para mayores dosis de $\mathrm{N}$ no se observan diferencias significativas en su productividad media. Asimismo, para el clon 433, el valor promedio de productividad obtenido con la dosis de 40 g.N $/ \mathrm{kg}$ es significativamente superior a los promedios obtenidos con dosis inferiores. Así, los valores promedios de productividad considerados mejores (significativamente superiores pero con menores dosis de g.N/kg) para los clones 103, 105, H77 y 433 fueron 14,5 (0 g.N/kg), 16,3 (36 g.N/kg), 12,5 (28 g.N/kg) y 13,5 (40 g.N/kg) 


\begin{tabular}{|c|c|c|c|c|}
\hline Dosis de Nitrógeno & \multirow{2}{*}{ Clon 103} & \multirow{2}{*}{ Clon 105} & \multirow{2}{*}{ Clon H77 } & \multirow{2}{*}{ Clon 433} \\
\hline g.N/kg & & & & \\
\hline 0 & $14,5 \mathrm{a}$ & $12,6 \mathrm{a}$ & $9,9 \mathrm{a}$ & $9,2 \mathrm{a}$ \\
\hline 28 & $15,4 \mathrm{a}$ & $13,0 \mathrm{a}$ & $12,5 \mathrm{ab}$ & $11,4 \mathrm{ab}$ \\
\hline 32 & $15,9 \mathrm{a}$ & $14,6 \mathrm{ab}$ & $12,9 \mathrm{ab}$ & $11,5 \mathrm{ab}$ \\
\hline 36 & $15,9 \mathrm{a}$ & $16,3 \mathrm{abc}$ & $12,9 \mathrm{ab}$ & $11,8 \mathrm{ab}$ \\
\hline 40 & $15,5 \mathrm{a}$ & $16,5 \mathrm{abc}$ & $12,9 \mathrm{ab}$ & $13,5 \mathrm{abc}$ \\
\hline
\end{tabular}

Letras diferentes indican diferencias significativas.

Cuadro 2. Promedios de producción de estaquillas de los 4 clones de Eucalyptus $\mathrm{x}$ urograndis para las 5 diferentes dosis de nitrógeno.

\begin{tabular}{|c|c|c|c|c|c|c|}
\hline 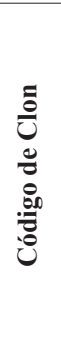 & 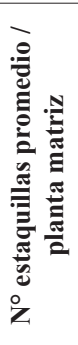 & 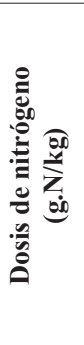 & 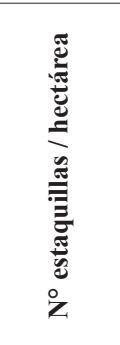 & $\begin{array}{l}85 \% \text { de eficiencia de } \\
\text { enraizamiento, según } \\
\text { Quispe (2017) }\end{array}$ & 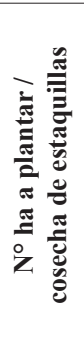 & 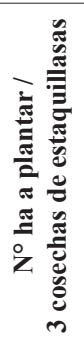 \\
\hline 103 & 14,5 & 0 & 96657,0 & 82158,5 & 74,0 & 221,9 \\
\hline 105 & 16,3 & 36 & 108655,8 & 92357,4 & 83,1 & 249,4 \\
\hline H77 & 12,5 & 28 & 83325,0 & 70826,3 & 63,8 & 191,3 \\
\hline 433 & 13,5 & 40 & 89991,0 & 76492,4 & 68,9 & 206,6 \\
\hline
\end{tabular}

Cuadro 3. Producción promedio de estaquillas de 4 clones de Eucalyptus $\mathrm{x}$ urograndis por hectárea de jardín clonal y número de hectáreas a plantar por cosecha de estaquillas.

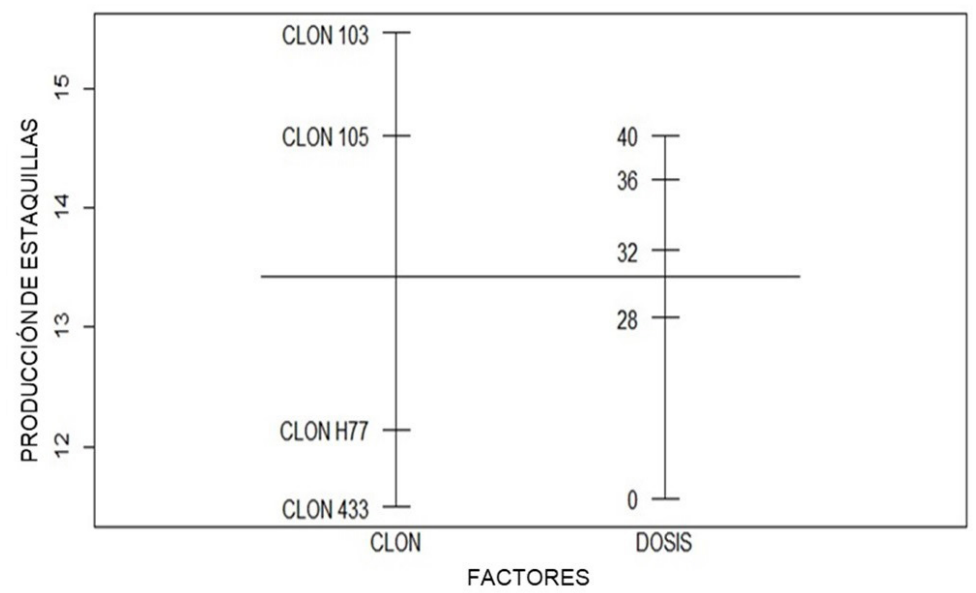

Figura 2. Efectos principales de los clones y las dosis de nitrógeno en la producción promedio de estaquillas. 
Efecto de dosis de nitrógeno sobre la producción de estaquillas de Eucalyptus grandis $\times$ E. urophylla en jardín clonal

\begin{tabular}{|c|c|c|c|c|c|c|}
\hline \multicolumn{7}{|c|}{ Respuesta: Productividad de estaquillas } \\
\hline & Df & Sum Sq & Mean Sq & F value & $\operatorname{Pr}(>\mathbf{F})$ & \\
\hline Clon & 3 & 217,883 & 72,628 & 253,0742 & $<2,2 \mathrm{e}-16$ & $* * *$ \\
\hline Dosis & 4 & 90,406 & 22,601 & 78,7559 & $<2,2 \mathrm{e}-16$ & $* * *$ \\
\hline Clon:Dosis & 12 & 29,463 & 2,455 & 8,5554 & $3,63 \mathrm{E}-09$ & $* * *$ \\
\hline Residuales & 60 & 17,219 & 0,287 & & & \\
\hline \multicolumn{7}{|c|}{ 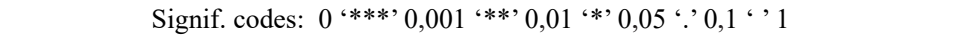 } \\
\hline
\end{tabular}

Cuadro 4. Análisis de varianza de la productividad de estaquillas del jardín clonal Eucalyptus $\mathrm{x}$ urograndis en el programa R 4.0.2.

estaquillas respectivamente (Cuadro 2), con un valor promedio general para estos clones y dosis de 14,2 estaquillas. Lo anterior se valida mediante la prueba comparativa de medias de Tukey con un nivel de confianza de $95 \%$ de probabilidad $(p \leq 0,05)$.

La mayor razón de rendimiento en hectáreas de jardín clonal/plantación fue de 1:83,1 para el clon 105 combinado con la dosis de 36 g.N/ $\mathrm{kg}$, es decir, por cada cosecha de estaquillas se puede realizar 83,1 ha de plantaciones forestales, considerando un $85 \%$ de eficiencia en el enraizamiento. Así, teniendo en cuenta las anteriores consideraciones, el clon 103 alcanzó una razón de rendimiento de 1:74,0 para la dosis 0 g.N $/ \mathrm{kg}$, seguida por el clon 433 con una razón de rendimiento de 1:68,9 para una dosis de 40 g.N $/ k g$ y finalmente el clon H77 con una razón de rendimiento de 1:63,8 para una dosis de 28 g.N/kg (Cuadro 3).

\section{Influencia de los clones y las dosis de nitróge- no en la productividad de estaquillas}

Se obtuvo diferencias significativas $(p \leq 0,05)$ en la productividad promedio de estaquillas respecto al efecto de los factores principales: clones y las diferentes dosis de nitrógeno aplicadas en las parcelas de evaluación. El clon 103 presentó mayor influencia en la producción promedio de estaquillas, seguido por el clon 105. En cuanto a las dosis de nitrógeno, el tratamiento con 40 g.N/kg presentó mayor efecto promedio en la producción (Figura 2).

Se observó variabilidad del efecto combinado de ambos factores sobre la producción promedio de estaquillas (Figura 3), con un alto nivel de significancia $(p \leq 0,05)$, según el análisis de varianza (ANVA) (Cuadro 4).

\section{Intervalo de cosecha de estaquillas}

Considerando la longitud de las estaquillas, se realizaron cuatro evaluaciones consecutivas para determinar los intervalos de cosecha en cada uno de los clones instalados en el jardín clonal (Figura 4), obteniéndose un intervalo general promedio de cosecha de estaquillas de 14,25 días, entre un mínimo de 13 días para el clon 433 y un máximo de 15 días para el clon 105, estos resultados se detallan en el Cuadro 5.

\section{Discusión}

\section{Productividad de estaquillas}

Las productividades obtenidas para todos los clones evaluados fueron superiores respecto a los registrados por Carvalho et al. (1991), citado por Higashi et al. (2000). En el cual, para el mismo género y en un intervalo de tiempo de entre 30 a 45 días obtuvieron 25 estaquillas, siendo estas inferiores a las 42,6 estaquillas obtenidas en promedio para los cuatro clones a nivel de jardín clonal en la presente investigación, considerando una productividad promedio general para los cuatro clones de 14,2 estaquillas y tres cosechas consecutivas con intervalos de 15 días. En contraste a otras técnicas de macro propagación, estudios de productividad realizados a nivel de minijardín clonal por Titon (2001) para un intervalo de 15 días, registró productividades promedias 


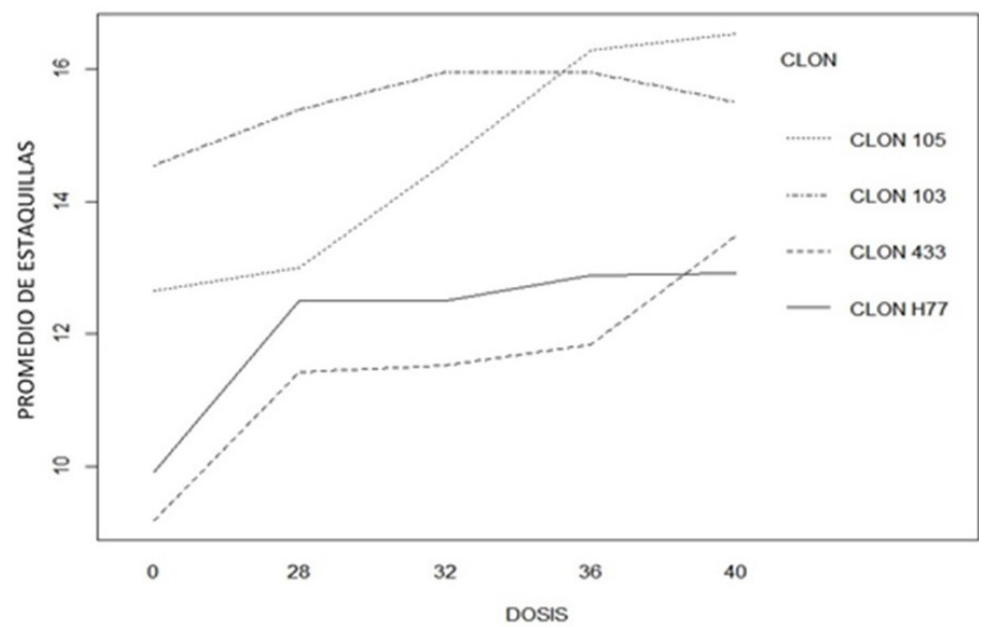

Figura 3. Variación del efecto combinado de ambos factores sobre la producción promedio de estaquillas.

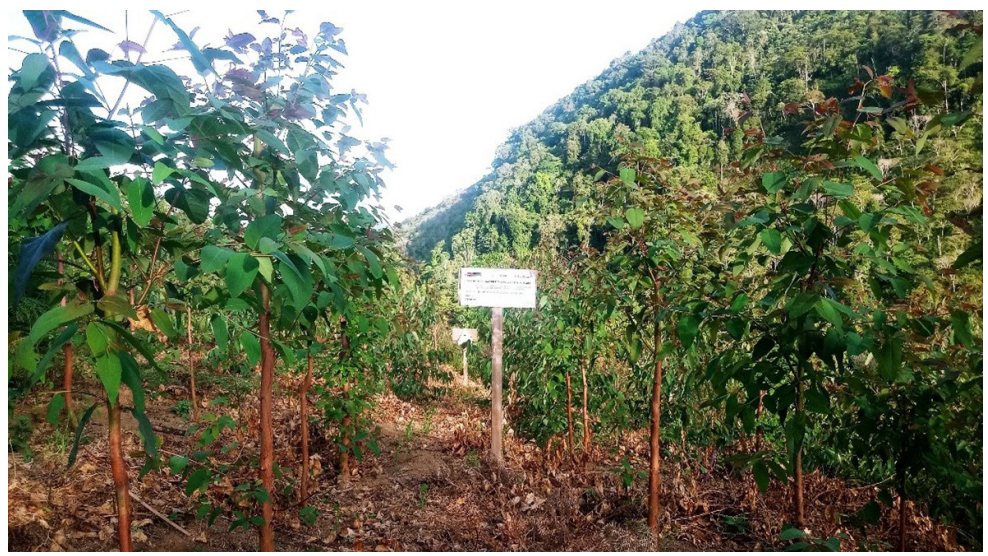

Figura 4. Jardín clonal de Eucalyptus $\mathrm{x}$ urograndis en Satipo, Junín.

de 11,5 miniestaquillas por planta, resultandos menores a las 14,2 estaquillas conseguidas en promedio en este estudio para el mismo intervalo de tiempo. Sin embargo, la calidad del material vegetativo producido a nivel de minijardín clonal es considerablemente superior en homogeneidad y concentración nutricional respecto a lo producido en un jardín clonal (Silveira et al. 1999, citado por Higashi et al. 2002).

En relación a la razón de rendimiento en hectáreas de jardín clonal/plantaciones, se con- siguió un máximo valor de 1:83,1 con el clon 105; estando este resultado cercano a la razón de rendimiento de 1:100 registrado por Campinhos et al. (1983). Sin embargo, las razones de rendimiento alcanzadas en este estudio, están muy por debajo de la razón de rendimiento registrado por Carvalho et al. (1991), citado por Higashi et al. (2000) la cual fue de 1:525, debiéndose principalmente al aumento de la densidad de plantío en el jardín clonal. El número de hectáreas de plantaciones forestales a instalarse es directamente proporcional al 
número de cosechas de estaquillas realizadas, así, por ejemplo, para el clon 105 si se realiza 3 cosechas de estaquillas, el número de hectáreas de plantaciones forestales a instalar será de 249,3; dicho cálculo se muestra en el Cuadro 3.

\section{Influencia de los clones y las dosis de nitróge- no en la productividad de estaquillas}

El análisis estadístico ANVA, arrojó influencia significativa para los factores tipo de clon y dosis de nitrógeno, así como también para el efecto combinado de los mismos sobre la producción de estaquillas.

Los clones 103 y 105 mostraron mayor predisposición para la producción de estaquillas respecto a los clones H77 y 433 para un mismo número de cosechas. Según Assis (1997), citado por Titon (2001), las variaciones genotípicas influyen en el proceso de producción de estaquillas, en el enraizamiento y en el desarrollo de las plantas. Asimismo, según López et al. (2016) en estudios realizados en un mismo sitio las variaciones genotípicas podrían estar sobreestimadas, por lo que sería conveniente realizar estudios similares a nivel de jardín clonal en otros sitios y en mayores periodos de evaluación para contar con mejores estimaciones de productividad.

Los clones estudiados presentaron comportamiento diferenciado para la producción de estaquillas en relación a las diferentes dosis de nitrógeno empleado, conforme lo señalado por Higashi et al. (2000) (Figura 3). Asimis-

\begin{tabular}{|c|c|c|c|c|c|c|}
\hline \multirow{2}{*}{$\begin{array}{c}\text { Código } \\
\text { Clonn }\end{array}$} & \multicolumn{7}{|c|}{$\begin{array}{c}\text { No de cosechas de } \\
\text { estaquillas }\end{array}$} \\
\cline { 2 - 7 } & $\mathbf{0}$ & $\mathbf{1}$ & $\mathbf{2}$ & $\mathbf{3}$ & $\mathbf{4}$ & $\overline{\mathbf{X}}$ \\
\cline { 2 - 7 } & \multicolumn{7}{|c|}{ Intervalo de cosecha } \\
(Días) \\
\hline 103 & - & 13 & 14 & 15 & 16 & 14,5 \\
\hline 105 & - & 15 & 15 & 15 & 15 & 15,0 \\
\hline H77 & - & 14 & 14 & 15 & 15 & 14,5 \\
\hline 433 & - & 13 & 13 & 13 & 13 & 13,0 \\
\hline
\end{tabular}

Cuadro 5. Promedio de intervalo para la cosecha de estaquillas de Eucalyptus $\mathrm{x}$ urograndis a nivel de jardín clonal. mo, se observó que cuanto mayor es la dosis de nitrógeno la producción de estaquillas se incrementa, coincidiendo con lo reportado por da Rosa et al. (2009). Sin embargo, estos incrementos no fueron significativos para todos los casos, así, por ejemplo, la tasa de producción de estaquillas del clon 103 con la dosis de 0 g.N/kg no mostró variación significativa $(p>$ $0,05)$ respecto a las otras dosis de nitrógeno. En contraste, el clon 105 presentó un incremento en su producción notablemente relacionada al incremento de la dosis de nitrógeno.

Asimismo, el efecto combinado de los factores muestra influencia significativa $(p \leq 0,05)$ sobre la producción de estaquillas a nivel de jardín clonal, existiendo respuesta diferenciada para cada clon de acuerdo con cada dosis de nitrógeno aplicado, coincidiendo con da Cunha et al. (2009), quien a su vez concluye que las exigencias nutricionales son de modo genotipo-dependiente.

Además, para determinar las combinaciones adecuadas clon:dosis se debe considerar también la eficiencia de las estaquillas en enraizamiento a nivel de invernadero, ya que según Terbulino et al. (2015) las dosis de nitrógeno influyen significativamente en el porcentaje

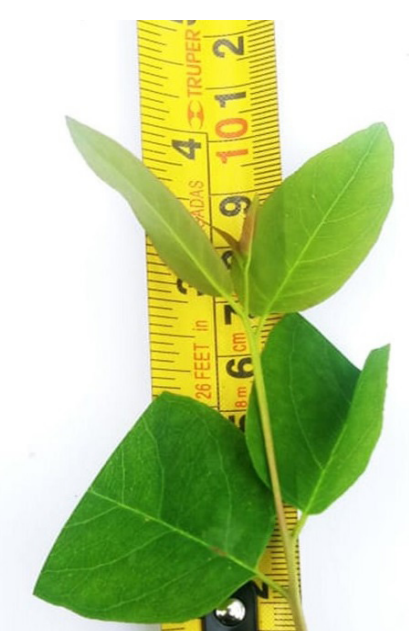

Figura 5. Número de hojas y tamaño de estaquilla de Eucalyptus $\mathrm{x}$ urograndis considerada adecuada para su colecta. 
de enraizamiento de miniestaquillas. Además, Cantarella (2007) menciona que el nitrógeno es responsable de las principales reacciones bioquímicas dentro de los vegetales, estando directamente relacionado con la capacidad fotosintética de las plantas.

\section{Intervalo óptimo de cosecha de estaquillas}

Los resultados muestran una estrecha relación con los parámetros de longitud y número de hojas acorde con lo mencionado por Terbulino et al. (2015). Así, la longitud promedio de estaquillas de 9,7 cm alcanzados en un intervalo promedio de 14,25 días para los cuatro clones estudiados (Figura 5), coinciden con lo obtenido por Paula (2000) a nivel de jardín clonal. Además, el intervalo de cosecha obtenido en esta investigación resultó menor que lo registrado en campo por Carvalho et al. (1991), citado por Higashi et al. (2000) y Terbulino et al. (2015), los cuales consiguieron intervalos entre 30 y 45 días.

Por último, las observaciones indican que periodos de precipitación prolongados aceleran el crecimiento en tamaño de las estaquillas y reducen el intervalo de cosecha de las mismas. De la misma manera, períodos de sequía prolongados pueden retrasar el crecimiento de las estaquillas e incrementar el intervalo de cosecha.

\section{Conclusiones}

La productividad de estaquillas a nivel de jardín clonal presentó influencia diferenciada por las dosis de $\mathrm{N}$ sobre los diferentes tipos de clones. Sin embargo, para el clon 103 se observó que esta relación no es determinante para la elección de la dosis de $\mathrm{N}$ a aplicar. Además, el clon 105 de Eucalyptus x urograndis presentó mayor productividad promedio de estaquillas a nivel de jardín clonal para las dosis de 36 y 40 g.N $/ \mathrm{kg}$, las cuales a su vez no presentaron diferencias significativas entre sí.

El intervalo promedio determinado para la cosecha de estaquillas a nivel de jardín clonal fue de 14,25 días, estando en función de la velocidad de crecimiento de las estaquillas en las plantas matrices del jardín clonal a campo abierto e influenciadas por las condiciones ambientales del sitio.

\section{Agradecimientos}

El presente trabajo se ha realizado gracias al financiamiento del Programa de Desarrollo Forestal Sostenible, Inclusivo y Competitivo en la Amazonía peruana del SERFOR. Asimismo, los autores agradecen a Julio Ugarte por su guía durante el desarrollo del presente trabajo de investigación, a los revisores anónimos y al editor de la RFP por sus valiosas sugerencias para mejorar el artículo..

\section{Bibliografía}

Badilla, Y; Murillo, O. 2005. Establecimiento de jardines clonales (en línea). Kurú: Revista Forestal 2(6):1-4. Consultado 18 jul. 2018. Disponible en https://dialnet.unirioja.es/descarga/ articulo/5123229.pdf

Campinhos, E; Ikemori, YK. 1983. Nova técnica para a produção de mudas de essências florestais (en línea). Instituto de Pesquisas e Estudos Florestais (23):43-46. Consultado 18 jul. 2018. Disponible en http://www.ipef.br/publicacoes/scientia/nr23/cap07.pdf

Cantarella, H. 2007. Nitrogênio. In Novais, RF, Alvarez, V, De Barros, N, Fontes, R, Cantarutti, R, Neves, J (eds.). Fertilidade do solo. Viçosa, Brasil, Sociedade Brasileira de Ciência do Solo. p. 375-470.

da Cunha, ACMCM; de Paiva, HN; de Barros, NF; Leite, HG; Leite, FP. 2009. Relação do estado nutricional de minicepas com o enraizamento de miniestacas de eucalipto. Revista Brasileira de Ciência do Solo 33(3):591599. DOI: http://dx.doi.org/10.1590/S0100$\underline{06832009000300012}$

da Rosa, LS; Wendling, I; Grossi, F; Reissmann, CB. 2009. Efeito da dose de nitrogênio e de formulações de substratos na miniestaquia de Eucalyptus dunnii Maiden. Revista Árvore 33(6):1025-1035.

FAO (Organización de las Naciones Unidas para la Alimentación y la Agricultura). 2003. 
World agriculture: towards 2015/2030 an FAO perspective (en línea). Bruinsma, J (ed.). Roma, Italia, Earthscan. 432 p. Consultado 02 ago. 2020. Disponible en http://www.fao.org/3/ a-y4252e.pdf

FONDEBOSQUE (Fondo de Promoción del Desarrollo Forestal). s.f.. Manejo en el primer año de una reforestación exitosa: tríptico informativo. Lima, Perú, s.e.. (2 p.).

Higashi, EN; Silveira, R; Gonçalves, AN. 2000. Propagação vegetativa de Eucalyptus: princípios básicos e a sua evolução no Brasil (en línea). São Paulo, Brasil, IPEF. 11 p. Circular Técnica n. ${ }^{\circ} 192$. Consultado 18 jul. 2018. Disponible en http://www.ipef.br/publicacoes/ ctecnica/nr192.pdf

Higashi, NE; Silveira, R; Gonçalves, AN. 2002. Nutrição e adubação em minijardim clonal hidropônico de Eucalyptus (en línea). São Paulo, Brasil, IPEF. 21 p. Circular Técnica n. ${ }^{\circ} 194$. Consultado 18 jul. 2018. Disponible en http:// www.ipef.br/publicacoes/ctecnica/nr194.pdf

López, JA; Harrand, L; Marcó, MA; López, AJ. 2016. Variación genética de clones híbridos de Eucalyptus (en línea). Quebracho 24(1):5-17. Consultado 29 jul. 2020. Disponible en https:// www.redalyc.org/jatsRepo/481/48163556005/ html/index.html

Martínez, R; Azpiroz, H; Rodríguez, JL; Cetina, VM; Gutiérrez, MA; Castellanos, JS. 2005. Micropropagación clonal in vitro en Eucalyptus grandis y E. urophylla. Ra Ximhai 1(1): 111-130.

Paula, TA. 2000. Efeito do potássio sobre a produção e enraizamento de estacas de Eucalyptus: reunión de fertilidad de suelos y nutrición de plantas (CD-ROM). Santa María, Brasil, 1 video, son., color.

Quiroz, I; García, E; González, M; Chung, P; Soto, H. 2009. Vivero forestal: producción de plantas nativas a raíz cubierta (en línea). Concepción, Chile, INFOR. 128p. Consultado 20 jul. 2020. Disponible en https://issuu.com/ helicongus/docs/produccion de plantas nativas a rai

Quispe, A. 2017. Estudio de las cadenas productivas y estrategias para la promoción de plantaciones forestales con fines comerciales y sostenibles en el Perú. Lima, Perú, SERFOR/ CAF. 257 p. Informe técnico s.n.. Programa de Desarrollo Forestal Sostenible, Inclusivo y Competitivo en la Amazonía Peruana.

Rocha, JHT; Backes, C; Borelli, K; Prieto, MR; Santos, AJM; Godinho, T. 2015. Produtividade do minijardim e qualidade de miniestacas de um clone híbrido de Eucalyptus grandis x Eucalyptus urophylla (I-224) em função de doses de nitrogênio (en línea). Ciencia Florestal 25(2):273-279. Consultado 12 jun. 2019. Disponible en https://www.scielo.br/pdf/cflo/ v25n2/0103-9954-cflo-25-02-00273.pdf

Sánchez, M. 2018. Forestación en Argentina: un buen negocio para el productor y el gobierno (en línea). In Fórum Forestal Internacional: "Gestión de negocios de las plantaciones forestales” (2018, Lima, Perú). CIP (Colegio de Ingenieros del Perú); SERFOR; Arborizaciones EIRL. Conferencias magistrales. Lima, Perú, s.e.. 81 p. Consultado 24 jul. 2018. Disponible en http://arborizaciones.com $/ \mathrm{mod} /$ folder/view.php?id=110

SERFOR (Servicio Nacional Forestal y de Fauna Silvestre). 2016. Estado Situacional de los viveros forestales en los departamentos de Ancash, Junín, Pasco, Huánuco, Apurímac y Ucayali. Lima, Perú, s.e.107 p. Programa Nacional de Promoción de Plantaciones Forestales Comerciales.

SERFOR (Servicio Nacional Forestal y de Fauna Silvestre). 2019a. Plan nacional de promoción de plantaciones forestales comerciales. Lima, Perú, SERFOR/CAF. 126 p. Programa de Desarrollo Forestal Sostenible, Inclusivo y Competitivo en la Amazonía Peruana. Preliminar.

SERFOR (Servicio Nacional Forestal y de Fauna Silvestre). 2019b. Anuario Forestal y de Fauna Silvestre 2017 (en línea). Lima, Perú, s.e. 124 p. Consultado 12 jun. 2019. Disponible en https://www.serfor.gob.pe/wp-content/uploads/2019/01/Anuario-FFS-2017.pdf

Tello, J; Tello, E. 2016. Clonación del eucalipto y bolaina. Lima, Perú, s.e. 132p. Proyecto "Selección y propagación asexual de candidatos 
de árboles plus de Guazuma crinita (bolaina) y Eucalyptus grandis $\mathrm{x}$ Eucalyptus urophylla (eucalipto urograndis) para el desarrollo de plantaciones forestales de alta productividad en áreas degradadas de la provincia de Oxapampa y la Selva Central del Perú".

Titon, M. 2001. Propagação clonal de Eucalyptus grandis por miniestaquia e microestaquia (en línea). Tesis MSc. Minas Gerais, Brasil, UFV. Consultado 20 jul. 2020. Disponible en https:// www.locus.ufv.br/handle/123456789/10899 